\title{
Higher Loop String Cosmology with Moduli and Antisymmetric Tensor Field
}

\author{
Aram A. Saharian \\ Department of Physics, Yerevan State University \\ 1 Alex Manoogian St., 375049 Yerevan, Armenia
}

\begin{abstract}
The classical evolution of a homogeneous cosmological model is investigated within the framework of low - energy effective string gravity with higher genus corrections. Various conformal frames are considered. For the general case of correction functions in the Lagrangian we give the cosmological solutions with arbitrary curvature and dilaton, modulus and Kalb-Ramond fields. They generalize previously known tree level solutions. The features of the solutions are discussed.
\end{abstract}

PACS Number(s): 98.80.Bp, 98.80.Cq, 98.80.Hw 


\section{Introduction}

Recently there has been a dramatic new development in the framework of superstring and supersymmetric Yang-Mills theories, which leads to the remarkable progress in understanding the non-perturbative aspects of these theories ( for recent reviews, see [1]-[5] ). Various duality symmetries play an essential role to compute strong coupling phases. One of the main consequences of string dualities is that the five apparently distinct superstring theories are actually related by duality transformations. In particular the strong coupling physics of certain superstring theories may be reformulated as the weak coupling physics of dual string theories ( $\mathrm{S}$ - duality). Similar property, known as $\mathrm{T}$ - duality, takes place with respect to length scales. One interesting example is the duality in six dimensions between heterotic strings compactified on 4-torus and type IIA superstring compactified to K3. Moreover, recent results indicate that different types of string models correspond to different perturbative expansions of the same underlying theory, the so - called M- theory [6], whose low-energy limit is eleven dimensional supergravity.

However, since beyond the first quantized framework of the Polyakov path integral, our knowledge of string theory is sorely lacking, it is very complicated to get definite results for quantum gravitational effects in framework of these theory. Therefore the investigation of stringy effects on the basis of low-energy effective theory becomes important. The presence of additional degrees of freedom (dilaton, axion etc.) essentially differs such a theory from General Relativity (GR). In particular, within the framework of such an approach a number of interesting results are obtained in black hole physics (see, for example [0]-[11] and references therein). Another area of investigations of stringy effects is the early cosmology. The cosmological consequences of string theory and their possible manifestations are important both for observational verification of theory and for the resolution of a number of problems in modern cosmology. Increasingly growing number of works in this direction (see [12- 63 and references therein) has resulted to the formation of a new area of cosmological investigations - String Cosmology. Recently, various cosmological models with dilaton and antisymmetric tensor field have been investigated to leading order in string tension as well as with higher-curvature corrections. In most cases the universe evolves toward strong coupling region where quantum loop corrections become important and the tree-level effective action can not reliably describe the dynamics. This motivates the consideration of models with loop corrections. Another motivation is related to the fact, that the string loop effects may naturally generate different nonmonotonic coupling functions in the effective action and consequently a relaxation mechanism (Damour - Polyakov mechanism) [31] by which various moduli fields are attracted towards their present vacuum expectation values. In particular, in 53] it was shown that an inflationary era within Damour - Polyakov mechanism could solve the Polonyi moduli problem.

Recently, an interesting alternative to the standard inflationary universe, moti- 
vated by the scale factor duality [18, 25] of the string effective action has been developed in [18, [27] and is known as pre-big bang inflation. According to this scenario the universe starts from string perturbative vacuum when the string dilaton is deep in the weak coupling region and curvature is small. Because of the instability of this vacuum the universe inflates to a stage with strong couplings and high curvature. It is assumed that after such an inflationary phase driven by dilaton kinetic energy, a branch change occurs to the standard radiation-dominated era (about the possibilities of such an exit see [33]-38]). The necessary conditions for successful exit are related to the violations of energy conditions appearing in Hawking - Penrose singularity theorems of GR [36], 38]. Loop corrections together with higher curvature terms are natural sources satisfying this requirement.

In the present paper we investigate multidimensional cosmological models with variable dilaton in the framework of low-energy effective string gravity with higher genus corrections. As an additional source a set of Kalb-Ramond and modulus fields is considered. In section 2 the structure of corresponding effective action and various conformal frames are discussed. The most important frames among these ones are string Einstein and Jordan frames. In section 3 the corresponding field equations are derived in general conformal frame and $D$-dimensional homogeneous anisotropic cosmological model is considered. The equations of model take the simplest form in Einstein frame (section 4). In particular, in this frame it is easy to find vacuum solutions with Ricci - flat subspaces. Further, the anisotropic solutions are considered with a set of zero potential scalar fields as a nongravitational source. At $D=4$ the Kalb - Ramond field can be reduced to scalar field (axion) as well. However for the axion, unlike the case of usual scalar fields, at tree level the Einstein and Jordan frames do not coincide. The set of scalar fields and Kalb - Ramond field is equivalent to an additional source with the extreme hard equation of state. For such a source the equations describing the evolution of cosmological scale factors in Einstein frame decouple from the scalar field equation. This allows to find the general solution for anisotropic model with Ricci - flat subspaces. In section 5 the isotropic model with curved space is considered. As in the previous case the extreme hard equation of state allows us to find the general solution for the set of scalar fields and Kalb - Ramond field. It generalizes previously known tree level solutions.

\section{$2 \quad$ String effective action and conformal frames}

Perturbative string theory contains two parameters: the string tension $\alpha$ ' of inverse mass squared dimension ( $h=c=1$ ) and dimensionless string coupling constant $g_{s}$. The first of them determines the length scale in the theory and controls stringy effects: at $\alpha^{\prime} \rightarrow 0$ the theory becomes the effective field theory. The second parameter $g_{s}$ controls the quantum corrections and is the parameter of loop expansion. At lowest 
order in string tension the effective action can be written down as [64]- [70], [31]

$$
S=\int d^{D} x \sqrt{|\widetilde{G}|}\left[-\widetilde{F}_{R}(\varphi) \widetilde{R}-4 \widetilde{F}_{\varphi}(\varphi) \partial_{M} \varphi \widetilde{\partial}^{M} \varphi+\widetilde{L}_{m}\left(\varphi, \widetilde{G}_{M N}, \psi\right)\right]
$$

where $\varphi$ is the dilaton field, $\widetilde{R}$ denotes curvature scalar of $D$ - dimensional metric $\widetilde{G}_{M N}, \widetilde{L}_{m}$ - is the Lagrangian density of other fields, collectively noted by a symbol $\psi$ and, in general, depending on the metric and dilaton (see below). Hereafter the symbol $\sim$ above the letters specifies the quantities in string conformal frame, the metric of which is the metric of corresponding $\sigma$-model. Note that the string coupling is given by the vacuum expectation value of the dilaton field: $g_{s}=\left\langle e^{2 \varphi}\right\rangle$. In the action (2.1) the most important special cases of Lagrangian are:

1) The antisymmetric Kalb-Ramond field $B_{M N}$ with strength $H_{M N P}=3 \partial_{[P} B_{M N]}$

$$
\widetilde{L}_{m}=\frac{1}{12} \widetilde{F}_{H}(\varphi) \widetilde{H}^{2}, \quad \widetilde{H}^{2}=H_{M N P} \widetilde{H}^{M N P}
$$

2) Gauge field with strength $F_{i M N}^{a}$ :

$$
\widetilde{L}_{m}=\sum_{i, a} \frac{1}{4 g_{i}^{2}} \widetilde{F}_{F}(\varphi) F_{i M N}^{a} \widetilde{F}_{i}^{a M N}
$$

where the index $i$ labels the various simple components of the gauge group, and index $a$ spans the corresponding adjoint representations.

3) Dilaton potential

$$
\widetilde{L}_{m}=-\tilde{V}(\varphi)
$$

4) Modulus fields $\psi_{i}$, which are string modes associated with compactification of extra dimensions when $D<D_{c r}$ :

$$
\widetilde{L}_{m}=\sum_{i} \widetilde{F}_{\psi_{i}}(\varphi) \widetilde{G}^{M N} \partial_{M} \psi_{i} \partial_{N} \psi_{i}-U\left(\varphi, \psi_{i}\right)
$$

In the simplest cases moduli correspond to the radii of compact dimensions.

The potential terms in (2.4) and (2.5) are expected to be nonperturbative, related to the supersymmetry breaking in the theory. The functions $\widetilde{F}_{K}(\varphi), K=$ $R, \varphi, H, F, \psi_{i}$ receive string perturbative as well as nonperturbative corrections. For the heterotic string on perturbative level we can write the following expansion

$$
\widetilde{F}_{K}(\varphi)=e^{-2 \varphi}\left(1+\sum_{l=1}^{\infty} \widetilde{Z}_{K}^{(l)} e^{2 l \varphi}\right)
$$

where dimensionless coefficient $\widetilde{Z}_{K}^{(l)}$ - represents $l$ - loop contribution, and the parameter of loop expansion is $e^{2 \varphi}$ : each loop of the string diagrams gives the contribution $\sim e^{2 \varphi}$. At $\varphi \ll-1$ the system is in the weak coupling region and is described by 
tree approximation of the string diagrams. Note that for the type-I and type-II superstrings the kinetic terms of the $\mathrm{R}$ - $\mathrm{R}$ fields do not have dilaton dependence at tree level.

As already it was noted above, the action (2.1) is written in string conformal frame. However, depending on the choice of relevant measuring units, physical are various conformal frames (about the physical equivalence of various conformal frames see, for example, [20, 22, 28, 46]). For example, if the act of measurement has an electromagnetic character, in this process the metric of conformal frame is measured, in which the electromagnetic part of the action is independent on dilaton field (Jordan frame for electromagnetic field). From the point of view of comparison of a dynamical picture of evolution of specific model in various conformal frames it is convenient to consider general conformal frame, connected to string frame by transformation of $D$ - dimensional metric:

$$
G^{M N}=\Omega^{2}(\varphi) \widetilde{G}^{M N}
$$

with arbitrary rather smooth function $\Omega(\varphi)$. Up to divergent terms in new conformal frame the action (2.1) takes the form

$$
S=\int d^{D} x \sqrt{|G|}\left[-F_{R}(\varphi) R-4 F_{\varphi}(\varphi) \partial_{M} \varphi \partial^{M} \varphi+L_{m}\left(\varphi, G_{M N}, \psi\right)\right],
$$

where the following new functions are introduced

$$
\begin{aligned}
4 F_{\varphi}(\varphi) & =\Omega^{n-1}\left[n(n-1)\left(\Omega^{\prime} / \Omega\right)^{2} \widetilde{F}_{R}+2 n\left(\Omega^{\prime} / \Omega\right) \widetilde{F}_{R}^{\prime}+4 \widetilde{F}_{\varphi}\right] \\
F_{R}(\varphi) & =\Omega^{n-1} \widetilde{F}_{R}, \quad L_{m}\left(\varphi, G_{M N}, \psi\right)=\Omega^{D} \widetilde{L}_{m}\left(\varphi, \Omega^{2} G_{M N}, \psi\right),
\end{aligned}
$$

$n=D-1$ - is the number of spatial dimensions, and the prime denotes differentiation with respect to $\varphi$. The action (2.8) represents a generalized $D$ - dimensional scalar tensor theory with nongravitational Lagrangian which depends on the scalar field.

At tree level it is convenient to choose the conformal multiplier in (2.7) as

$$
\Omega^{2}(\varphi)=e^{c \varphi}
$$

with arbitrary constant $c$. The gravitational part of the corresponding action is now equivalent to $D$ - dimensional Jordan - Brans - Dicke theory with parameter [22], 46, 47.

$$
\omega=-1+\frac{8-(n-1) c}{[4-(n-1) c]^{2}} c
$$

In particular, in string frame $(c=0)$ we receive from here $\omega=-1$.

The one of the most important special cases of (2.8) is the action in Einstein (E-) frame, when the term with Ricci scalar has the standard canonical form. The following choice of the conformal factor corresponds to this frame

$$
\Omega^{2}(\varphi)=\widetilde{F}_{R}^{-2 /(n-1)}
$$


and the corresponding action takes the form

$$
S=\int d^{D} x \sqrt{|\bar{G}|}\left[-\bar{R}-4 \bar{F}_{\varphi}(\varphi) \partial_{M} \varphi \bar{\partial}^{M} \varphi+\bar{L}_{m}\left(\varphi, \bar{G}_{M N}, \psi\right)\right]
$$

where overbars indicate that he corresponding quantities are taken in the E-frame. Here we assumed, that $\widetilde{F}_{R}>0$. If this function is negative valued, the conformal factor can be chosen similarly (2.12) with the absolute value. Now in the corresponding action the Ricci scalar will enter with positive sign, and the corresponding gravitational constant will be negative. The function at the kinetic term of dilaton field is connected to the corresponding function of string frame by relation

$$
4 \bar{F}_{\varphi}(\varphi)=-\frac{n}{n-1}\left(\frac{\widetilde{F}_{R}^{\prime}}{\widetilde{F}_{R}}\right)^{2}+4 \frac{\widetilde{F}_{\varphi}}{\widetilde{F}_{R}} .
$$

For $\bar{F}_{\varphi}<0$ by introducing a new scalar field $\phi$ as

$$
d \phi=2 \sqrt{-\bar{F}_{\varphi}} d \varphi
$$

the kinetic term can be written in canonical form. Below we shall consider just this case. At tree level $\bar{F}_{\varphi}=-1 /(n-1)$ and the new scalar field is proportional to the dilaton:

$$
\phi=\frac{2}{\sqrt{n-1}} \varphi
$$

Another important conformal frame of scalar - tensor theories is the so-called Jordan frame, in which the nongravitational part of the action does not contain the scalar field. In this frame the laws of evolution of the nongravitational fields take the same form as in multidimensional GR. For the action (2.8) the Jordan frame, in general, can not be realized. Let us consider the important special case, when in (2.1) the dilaton dependence is factorized:

$$
\widetilde{L}_{m}\left(\varphi, \widetilde{G}_{M N}, \psi\right)=\widetilde{F}_{L}(\varphi) \widetilde{L}\left(\widetilde{G}_{M N}, \psi\right) .
$$

For these Lagrangians the Jordan frame exists, if the function $\widetilde{L}$ has a certain conformal weight $\beta$ :

$$
\widetilde{L}\left(\Omega^{2} G_{M N}, \psi\right)=\Omega^{2 \beta} \widetilde{L}\left(G_{M N}, \psi\right)
$$

In general conformal frame the corresponding function is determined from the last relation of (2.9) and has the form

$$
L_{m}=\Omega^{D+2 \beta} \widetilde{F}_{L}(\varphi) \widetilde{L}\left(G_{M N}, \psi\right)
$$

Now the choice of the conformal factor in accordance with

$$
\Omega^{D+2 \beta}=\left|\widetilde{F}_{L}(\varphi)\right|^{-1}
$$


leads to the Jordan frame with exception of the case $\beta=-D / 2$, when $\widetilde{L}_{m}$ is conformal invariant. For examples considered above the parameter $\beta$ has the following values: $\beta=-3$ for Kalb - Ramond field, $\beta=-2$ for gauge field, $\beta=0$ for dilaton potential. The Lagrangian (2.5) has a certain conformal weight $(\beta=-1)$ if the potential terms are absent and the condition (2.17) is fulfilled if the functions at kinetic terms are universal. Note that for the R-R fields the string and Jordan frames coincide at tree level.

The next conformal frame, useful for calculations of quantum gravity effects in background field method (see for example [71]) is the frame with no dilaton kinetic term in the action, corresponding to $F_{\varphi}(\varphi)=0$ in (2.8). As it follows from relation (2.9) such a frame exists when $\bar{F}_{\varphi} \leq 0$.

\section{$3 \quad$ Field equations and cosmological model}

By introducing a new scalar field

$$
\Phi=F_{R}(\varphi)
$$

the action (2.8) can be written as

$$
S=\int d^{D} x \sqrt{|G|}\left[-\Phi R+\omega(\Phi) \partial_{M} \Phi \partial^{M} \Phi / \Phi+L\left(\Phi, G_{M N}, \psi\right)\right],
$$

where the following notation is introduced

$$
\omega(\Phi)=-4 F_{\varphi} \frac{F_{R}}{F_{R}^{\prime 2}}, \quad L\left(\Phi, G_{M N}, \psi\right)=L_{m}\left(\varphi(\Phi), G_{M N}, \psi\right)
$$

The theory defined by (3.2) is a generalized $D$ - dimensional scalar - tensor theory of gravity with nontrivial coupling of gravitational scalar to the nongravitational sector. The variation of this action leads to the following equations of motion for $G_{M N}$ and $\Phi$ (see also [72 for corresponding equations in scalar-tensor theories and [22, 46, 47] for corresponding tree level equations):

$$
\begin{aligned}
R_{M N}-\frac{1}{2} G_{M N} R= & \frac{1}{2 \Phi} T_{M N}+\frac{1}{\Phi}\left(D_{M} D_{N} \Phi-G_{M N} \square \Phi\right)+ \\
& +\frac{\omega}{\Phi^{2}}\left(\partial_{M} \Phi \partial_{N} \Phi-\frac{1}{2} G_{M N} \partial_{P} \Phi \partial^{P} \Phi\right), \\
\frac{2 \omega}{\Phi} \square \Phi+\partial_{P} \Phi \partial^{P}\left(\frac{\omega}{\Phi}\right)= & -R+\frac{1}{\sqrt{|G|}} \frac{\delta(L \sqrt{|G|})}{\delta \Phi},
\end{aligned}
$$

where $D_{M}$ denotes the covariant derivative by metric $G_{M N}, \square=G^{M N} D_{M} D_{N}$ is the covariant Dalambertian,

$$
T_{M N}=\frac{2}{\sqrt{|G|}} \frac{\delta(L \sqrt{|G|})}{\delta G^{M N}}
$$


is the energy - momentum tensor. Eliminating $R$ with the help of convolution of the first equation (3.4) these equations can be written down also as

$$
\begin{aligned}
R_{M N}-\frac{1}{2 \Phi}\left(T_{M N}-\frac{G_{M N}}{n-1} T\right)= & \Phi^{-1}\left(D_{M} D_{N} \Phi+\frac{G_{M N}}{n-1} \square \Phi\right) \\
& +\frac{\omega}{\Phi^{2}} \partial_{M} \Phi \partial_{N} \Phi, \\
2\left(\omega+\frac{n}{n-1}\right) \square \Phi+\partial^{P} \Phi \partial_{P} \omega= & \frac{1}{n-1}\left(T+\frac{n-1}{\left.\sqrt{|G|} \Phi \frac{\delta L \sqrt{|G|}}{\delta \Phi}\right) .}\right.
\end{aligned}
$$

As a consequence of dilaton dependence of Lagrangian $L$ the corresponding energymomentum tensor is acted upon by a dilaton gradient force:

$$
D_{M} T_{N}^{M}=-\frac{1}{\sqrt{|G|}} \frac{\delta L \sqrt{|G|}}{\delta \Phi} \partial_{N} \Phi .
$$

We shall consider $D$ - dimensional homogeneous cosmological model with spacetime structure $R \otimes M^{1} \otimes \ldots \otimes M^{P}$ and with the metric

$$
G_{M N}=\operatorname{diag}\left(N^{2}(t), \ldots,-R_{i}^{2}(t) g_{l m}^{(i)}, \ldots\right)
$$

where $M_{i}$ is a maximally symmetric $n_{i}$ dimensional space, $\sum n_{i}=n, g_{l m}^{(i)}$ is the metric in this space, $R_{i}(t)$ and $N$ are corresponding scale factors and lapse function. Functions $N(t)$ and $R_{i}(t)$,. are depending on conformal frame and are related to the corresponding quantities in string and $\mathrm{E}$ - frames through the transformations

$$
\begin{aligned}
& N(t)=\Omega^{-1}(\varphi) \widetilde{N}(t)=\bar{\Omega}^{-1} \bar{N}(t), \\
& R_{i}(t)=\Omega^{-1}(\varphi) \widetilde{R}_{i}(t)=\bar{\Omega}^{-1} \bar{R}_{i}(t), \quad \bar{\Omega} \equiv \Omega \widetilde{F}_{R}^{1 /(n-1)} .
\end{aligned}
$$

They relate the pictures of cosmological evolution in various frames. From the field equations it follows, that for the metric (3.9) the energy - momentum tensor is diagonal and can be represented as

$$
T_{N}^{M}=\operatorname{diag}\left(\varepsilon, \ldots,-\delta_{n}^{m} p_{i}, \ldots\right),
$$

where $\varepsilon$ is the energy density, $p_{i}$ is the effective pressure in the subspace $M^{i}$. If the Lagrangian does not depend on derivatives of the metric tensor, the values of these quantities in various conformal frames are related by

$$
T_{N}^{M}=\Omega^{D} \widetilde{T}_{N}^{M}=\bar{\Omega}^{D} \bar{T}_{N}^{M}
$$

Introducing the notation

$$
a_{i}=p_{i} / \varepsilon, \quad \alpha=\frac{1}{\varepsilon \sqrt{|G|}} \frac{\delta L \sqrt{|G|}}{\delta \varphi}, \quad \bar{a}=1-\sum_{i=1}^{p} n_{i} a_{i}
$$


The equations of cosmological model can be written down as

$$
\begin{aligned}
\dot{H}_{i}+y H_{i}-\delta_{i} \frac{b^{2}}{2} \frac{\dot{\Phi}}{\Phi} \omega+N^{2} k_{i} \frac{n_{i}-1}{R_{i}^{2}} & =\frac{N^{2}}{\Phi} b_{i} \varepsilon, \quad i=0,1, \ldots, p \\
\sum_{i, l=0}^{p} a_{i l} H_{i} H_{l}+\sum k_{i} n_{i} N^{2} \frac{n_{i}-1}{R_{i}^{2}} & =\frac{N^{2}}{\Phi} \varepsilon
\end{aligned}
$$

where the overdots denote time derivatives , $k_{i}=-1,0,1$ for subspaces with negative, zero and positive curvatures, correspondingly,

$$
\begin{aligned}
H_{i} & =\dot{R}_{i} / R_{i}, \quad i=0,1, \ldots, p, \quad R_{0} \equiv \Phi, \quad n_{0}=1 \\
y & =\sum_{i=0}^{p} n_{i} H_{i}-\dot{N} / N, \quad b^{2}=[\omega(n-1)+n]^{-1}=-\left[4(n-1) \bar{F}_{\varphi}\right]^{-1}\left(F_{R}^{\prime} / F_{R}\right)^{2} \\
b_{0} & =\frac{1}{2} b^{2}\left[\bar{a}+(n-1) \alpha \frac{F_{R}}{F_{R}^{\prime}}\right], \quad b_{i}=\frac{1}{2}\left(a_{i}+\frac{\bar{a}}{n-1}\right)-\frac{b_{0}}{n-1}, \quad i=1, \ldots, p \\
\delta_{0} & =1-n, \quad \delta_{1}=1, \quad a_{i l}=n_{i} n_{l}-n_{l} \delta_{i l}, \quad i=1, \ldots, p, \quad a_{00}=-\omega .
\end{aligned}
$$

At tree level and for the conformal factor (2.10) the function $b(\varphi)$ is constant

$$
b=\frac{c}{4}(n-1)-1, \quad F_{R}=e^{2 b \varphi} .
$$

The choice $N=1$ corresponds to the synchronous reference system in general conformal frame, and synchronous time coordinate is depend on particular frame. In the cosmological context the conservation equation (3.8) takes the form

$$
\dot{\varepsilon} / \varepsilon+\sum_{i=1}^{p} n_{i}\left(1+a_{i}\right) H_{i}+\alpha \dot{\varphi}=0,
$$

where the quantities $a_{i}$ and $\alpha$, in general, are functions of time. Note that in the Jordan frame $\alpha=0$. From (3.13) it follows, that $a_{i}$ are conformal invariant, and the quantities $\alpha$ in various conformal frames are connected by relations

$$
\alpha=\widetilde{\alpha}-\bar{a} \Omega^{\prime} / \Omega
$$

if the Lagrangian $\widetilde{L}_{m}$ does not depend on derivatives of the metric.

In the case of the equation of state with constant $a_{i}$ and for the function $\alpha$, depending only on dilaton field, the integration of equation (3.18) yields

$$
\varepsilon=\text { const } \cdot \exp \left(-\int \alpha(\varphi) d \varphi\right) \prod_{i=1}^{p} R_{i}^{-n_{i}\left(1+a_{i}\right)}
$$

In particular, for dust matter $a_{i}=0$ and we obtain $\varepsilon \sim 1 / V$, where $V$ is the volume of multidimensional space. 
As an example of an additional source we shall consider system of scalar fields $\psi_{i}$ with Lagrangian density (2.5) for the case of zero potential. Within the framework of homogeneous cosmological models assuming that $\psi_{i}=\psi_{i}(t)$ we obtain for corresponding energy density and pressure

$$
\varepsilon=p_{i}=L_{m}=\sum_{i} F_{\psi_{i}} \dot{\psi}_{i}^{2} / N^{2}
$$

and therefore

$$
a_{i}=1, \quad i=1, \ldots, p,
$$

that is the system is described by the extreme hard equation of state. The corresponding coefficients $b_{i}$ are determined from (3.16) and are equal

$$
b_{0}=\frac{F_{R}^{\prime}}{8 F_{R} \bar{F}_{\varphi}}\left(\frac{F_{R}^{\prime}}{F_{R}}-\alpha\right), \quad b_{i}=\frac{b_{0}}{1-n}, i=1, \ldots, p
$$

From the equation of motion for the field $\psi_{i}$

$$
\partial_{M}\left(\sqrt{|G|} F_{\psi_{i}} G^{M N} \partial_{N} \psi_{i}\right)=0,
$$

we now obtain

$$
\dot{\psi}_{i}=\frac{C_{i} N}{V F_{\psi_{i}}}, \quad V=\prod_{i=1}^{p} R_{i}^{n_{i}}
$$

where $C_{i}$ are integration constants. Hence the expressions for the energy density and function $\alpha$ take the form

$$
\varepsilon=V^{-2} \sum_{i} C_{i}^{2} F_{\psi_{i}}^{-1}, \quad \alpha=\frac{1}{V^{2} \varepsilon} \sum C_{i}^{2} F_{\psi_{i}}^{\prime} / F_{\psi_{i}}^{2} .=-\left[\ln \left(\varepsilon V^{2}\right)\right]^{\prime}
$$

Since the function $\alpha$ depends only on dilaton field, the last two relations can be derived also by starting directly with (3.20).

Another important example of an additional source is Kalb - Ramond field (see (2.2)). The corresponding equations of motion have the form

$$
\partial_{M}\left(\sqrt{|G|} F_{H} H^{M N P}\right)=0, \quad F_{H}=\Omega^{D-6} \widetilde{F}_{H} .
$$

In $D=4$ these equations are solved by the ansatz

$$
H^{M N P}=\frac{1}{\sqrt{|G|}} F_{H}^{-1} \varepsilon^{M N P Q} \partial_{Q} h,
$$

where $\varepsilon^{M N P Q}$ is completely antisymmetric 4-tensor, $h$ is the pseudoscalar field of axion. From the Bianci identity $\partial_{[Q} H_{M N P]}=0$ the equation of motion for the field $h$ follows:

$$
\square h-\partial^{M} h \partial_{M} F_{H} / F_{H}=0
$$


The corresponding Lagrangian density has the form

$$
L_{m}=F_{h} \partial^{M} h \partial_{M} h, \quad F_{h}=1 /\left(2 F_{H}\right)
$$

in general conformal frame related to the string one by transformation (2.7). Thus in this case the Kalb - Ramond field is equivalent to the pseudoscalar field $h$ and the corresponding formulas are a special case of the previous example. However it is necessary to note, that if for usual scalar fields in the string frame at tree level $\widetilde{F}_{\psi_{i}}=e^{-2 \varphi}$, for the heterotic string axion, as it follows from (3.30), $\widetilde{F}_{h}=e^{2 \varphi} / 2$ and for the type-I axion $\widetilde{F}_{h}=1 / 2$.

\section{Solutions with Ricci - flat subspaces}

The cosmological field equations are simplest in the Einstein metric. By introducing a new scalar field $\phi$ in accordance with (2.15), the system of equations (3.15) can be written as (here and below, to not complicate the formulas, we omit the overbars over the letters indicating the quantities in the $\mathrm{E}$ - frame; everywhere, where it is not especially noted, the $\mathrm{E}$ - frame is considered)

$$
\begin{aligned}
\dot{H}_{i}+\left(\sum_{i=1}^{p} n_{i} H_{i}-\dot{N} / N\right) H_{i} & =N^{2} b_{i} \varepsilon-N^{2} k_{i} \frac{n_{i}-1}{R_{i}^{2}}, \quad i=1, \ldots, p \\
\ddot{\phi}+\left(\sum_{i=1}^{p} n_{i} H_{i}-\dot{N} / N\right) \dot{\phi} & =\frac{1}{2} N^{2} \alpha_{\phi} \varepsilon \\
N^{2} \varepsilon+\dot{\phi}^{2}-\sum k_{i} n_{i} N^{2} \frac{n_{i}-1}{R_{i}^{2}} & =\sum_{i, l=1}^{p} a_{i l} H_{i} H_{l}
\end{aligned}
$$

where now in E - frame

$$
b_{i}=\frac{1}{2}\left(a_{i}+\frac{\bar{a}}{n-1}\right), \quad \alpha_{\phi}=\frac{1}{\varepsilon \sqrt{|G|}} \frac{\delta L \sqrt{|G|}}{\delta \phi}
$$

From the second equation of (4.1) it follows, that for the solutions with constant dilaton $\varphi=\varphi_{0}$, the values $\varphi_{0}$ have to be roots of the equation $\alpha(\varphi)=0$. The dilaton can be fixed both by dilaton potential, or by nontrivial dependence of Lagrangian of the other fields on dilaton (see, for example, (2.2) and (2.3)). The second way is known as Damour - Polyakov mechanism [31]. The solutions with constant dilaton coincide with corresponding solutions of multidimensional GR. Let us consider the behavior of the cosmological models in the neighborhood of these solutions for the case of a set of zero potential scalar fields as a nongravitational source (see (3.21)) (on the relaxation of scalar - tensor isotropic cosmological models to the solutions with 
constant scalar field see [73] and references therein). For such a source it is convenient to introduce a new variable $\tau$ in accordance with

$$
d \tau=\frac{N}{V} d t
$$

By taking into account (3.20) and (3.26) we obtain from the second equation (4.1) an evolution equation for $\phi$ as function of $\tau$ :

$$
\frac{d^{2} \phi}{d \tau^{2}}=-\frac{1}{2} \frac{d}{d \phi}\left(\varepsilon V^{2}\right)
$$

As it follows from here for a solution with constant scalar field $\phi=\phi_{0}$ the value $\phi_{0}$ has to be an extremum of the function $\varepsilon V^{2}$ :

$$
\frac{d}{d \phi}\left(\varepsilon V^{2}\right)=\frac{d}{d \phi} \sum_{i} \frac{C_{i}^{2}}{F_{\psi_{i}}}=0, \quad \phi=\phi_{0}
$$

The equation (4.4) can be thought of as describing a one dimensional dynamics of a particle with a potential term $V^{2} \varepsilon / 2$. The singular points of the corresponding autonomous dynamical system are determined by equation (4.5). On the phase plane $(\phi, d \phi / d t)$ these points are saddle points for $\omega_{0} \equiv\left(\frac{d^{2}}{d \phi^{2}} V^{2} \varepsilon\right)_{\phi=\phi_{0}}<0$ and center for $\omega_{0}>0$. For both these cases the dilaton dependence of the Lagrangian for modulus and antisymmetric tensor fields can not drive the cosmological model toward a state with constant dilaton. For the isotropic flat cosmological model this result follows also from the analysis of Ref. [73], [31] and is a consequence of the fact that for the source with equation of state $\varepsilon=p$ the corresponding friction term in the scalar field equation vanishes. Note that for the case $\omega_{0}>0$ in the neighborhood of singular point the trajectories on the phase plane $(\phi, d \phi / d t)$ are closed curves and the corresponding solutions have the form $\phi-\phi_{0} \sim \cos \left(\sqrt{\omega_{0}} \tau+\tau_{0}\right)$.

\subsection{Pure gravi - dilaton solutions}

We start with the simple case of Ricci - flat subspaces $\left(k_{i}=0\right)$ and zero nongravitational sources $\left(L_{m}=0\right)$. From the system (4.1) we find

$$
H=\text { const } \cdot N / V, \quad \dot{\phi}=\text { const } \cdot N / V
$$

Introducing $\mathrm{E}$ - frame synchronous time coordinate $t_{E}$ :

$$
d t_{E}=N(t) d t
$$

the solutions to cosmological equations can be written as

$$
H_{i}=\frac{H_{i 0}}{t_{E}-t_{0}}, \quad R_{i}=R_{i 0}\left|t_{E}-t_{0}\right|^{H_{i 0}}, \quad \sum_{i=1}^{p} n_{i} H_{i 0}=1
$$




$$
\phi=\phi_{10} \ln \left|t_{E}-t_{0}\right|+\phi_{0}, \quad \phi_{10}^{2}+\sum_{i=1}^{p} n_{i} H_{i 0}^{2}=1
$$

where $H_{i 0}, R_{i 0}, \phi_{0}, t_{0}$ - are integration constants. The last relation is a consequence of the constraint equation. Then $\phi_{10}=0$ from (4.8), (4.9) one obtains the multidimensional generalization of the Kasner solution of the $D$ - dimensional GR. Note that unlike the case of Kasner solution, the solution (4.8),(4.9) has an isotropic limit when one obtains $H_{10}=1 / n, \quad \phi_{10}= \pm \sqrt{1-1 / n}$.

For given functions $\widetilde{F}_{\varphi}(\varphi)$ and $\widetilde{F}_{R}(\varphi)$ the time dependence of dilaton field is determined from

$$
\phi_{10} \ln \left|t_{E}-t_{0}\right|=2 \int \sqrt{-\bar{F}_{\varphi}} d \varphi
$$

where the integrand can be expressed through the previous functions by the relation (2.14). Note that the behavior of dilaton field is always monotonic if the function $\bar{F}_{\varphi}$ does not change the sign. The corresponding solution in string frame can be found by transformation

$$
\widetilde{R}_{i}=\widetilde{F}_{R}^{1 /(1-n)} R_{i}, \quad d t_{s}=\widetilde{F}_{R}^{1 /(1-n)} d t_{E}
$$

where $t_{s}$ is string frame synchronous time coordinate. At tree level the solution of string frame takes the form 17, 18

$$
\begin{aligned}
\varphi-\varphi_{0} & =\varphi_{10} \ln \left|t_{s}-t_{s 0}\right|, \quad \widetilde{R}_{i}=\widetilde{R}_{i 0}\left|t_{s}-t_{s 0}\right|^{\widetilde{H}_{i 0}} \\
\sum_{i=1}^{p} n_{i} \widetilde{H}_{i 0} & =1+2 \varphi_{10}, \quad \sum_{i=1}^{p} n_{i} \widetilde{H}_{i 0}^{2}=1
\end{aligned}
$$

The same solution in general conformal frame can be found in 48]. The corresponding isotropic solution has the form

$$
\varphi=\varphi_{0}+\frac{1}{2}( \pm \sqrt{n}-1) \ln \left|t_{s}-t_{s 0}\right|, \quad H_{i}=\frac{ \pm 1}{\sqrt{n}\left(t_{s}-t_{s 0}\right)}
$$

In the case $t_{s}<t_{s 0}$ and for the lower sign, (4.13) describes superinflationary expansion, when the evolution of the universe starts at $t_{s}=-\infty$ and dilaton is deep in weak coupling region. Such an expansion is driven by kinetic energy of the dilaton field and corresponds to the pre-big bang phase of the mechanism for inflationary evolution proposed in [18], [27].

\subsection{Solutions with modulus and Kalb - Ramond fields}

In the equations (4.1) as an additional source we shall consider a set of scalar fields and Kalb - Ramond field:

$$
L_{m}=\sum_{i} F_{\psi_{i}} \partial^{M} \psi_{i} \partial_{M} \psi_{i}+\frac{1}{12} F_{H}(\varphi) H^{2}
$$


As it has been mentioned already, if we accept the ansatz (3.28), the Kalb - Ramond field is also reduced to the scalar field with Lagrangian (3.30) . In particular, in cosmological context we shall assume

$$
\varepsilon=p_{i}=L_{m}=\sum_{i}^{\prime} F_{\psi_{i}} \dot{\psi}_{i}^{2} / N^{2} \equiv \sum_{i} F_{\psi_{i}} \dot{\psi}_{i}^{2} / N^{2}+F_{h} \dot{h}^{2} / N^{2}
$$

and prime specifies the fact that the corresponding sum includes also the contribution of the axion field. For the functions $F_{\psi_{i}}$ in accordance with (2.7) and (2.12) we have in $\mathrm{E}$ - frame

$$
F_{\psi_{i}}=\widetilde{F}_{\psi_{i}} / \widetilde{F}_{R}, F_{h}=\left(2 \widetilde{F}_{H} \widetilde{F}_{R}\right)^{-1}
$$

It follows from here, that at tree level of the heterotic superstring, thanks to the universality of the functions $\widetilde{F}_{k}(\varphi)$ :

$$
F_{\psi_{i}}=1, \quad F_{h}=e^{4 \varphi} / 2
$$

For the type-I superstring we have $F_{h}=e^{2 \varphi} / 2$. Thus, for usual scalar fields (it concerns also to the dilaton field) at tree level the $\mathrm{E}$ - and Jordan frames coincide, while for axion field this is not the case. By taking into account these relations one obtains the following asymptotic behavior of potential term of (4.4) in weak coupling region:

$$
\varepsilon V^{2} / 2=\sum_{i}^{\prime} \frac{C_{i}^{2}}{2 F_{\psi_{i}}} \sim \frac{1}{2} \sum_{i} C_{i}^{2}+C_{h}^{2} e^{-4 \varphi}, \quad \varphi \ll-1
$$

and the contribution of the axion field dominates. It follows from (4.15), that for a source with the Lagrangian (4.14) all coefficients $a_{i}$ are equal to 1 , and therefore as we can see from (4.2) all coefficients $b_{i}$ are equal to zero. For these values and for Ricci flat subspaces the solution of the first equation (4.1) for scale factors in synchronous system of coordinates is still determined by the relation (4.8). From the constraint equation we now have

$$
\varepsilon+\dot{\phi}^{2}=A\left(t_{E}-t_{0}\right)^{-2}, \quad A \equiv 1-\sum_{i=1}^{p} n_{i} H_{i 0}^{2},
$$

(note that, if the all subspaces are expanding then the constant $A$ is positive) where the energy density is determined by the relation (see (3.26))

$$
\varepsilon=\frac{1}{V_{0}^{2}\left(t_{E}-t_{0}\right)^{2}} \sum_{i}^{\prime} \frac{C_{i}^{2}}{F_{\psi_{i}}}
$$

The substitution of this expression in (4.19) and integration of the obtained equation leads to the following result

$$
\ln \left|t_{E}-t_{0}\right|= \pm \int\left[A-\sum_{i} C_{i}^{2} V_{0}^{-2} / F_{\psi_{i}}\right]^{-1 / 2} d \phi
$$


Here the functions $F_{\psi_{i}}$ are related to the corresponding functions of the string frame by (4.16). Further for given function $\varphi\left(t_{E}\right)$ from the equations (3.25) one finds

$$
\psi_{i}=\int \frac{C_{i} d t_{E}}{V_{0} F \psi_{i}(\varphi)\left|t_{E}-t_{0}\right|}
$$

For the given coupling functions in (2.1) the formulas (4.8), (4.16), (4.21), (4.22) determine the evolution of the corresponding cosmological model in the E-frame. The string frame solutions can be found by transformations (4.11). Note that unlike the case of the E-frame, the solutions for string frame scale factors do not coincide with corresponding vacuum solutions.

As has been previously mentioned, for the solutions with constant dilaton $\varphi=\varphi_{0}$ one has $\alpha\left(\varphi_{0}\right)=0$. For such a solution together with (4.5) we have the following relation between the integration constants

$$
\sum_{i}^{\prime} C_{i}^{2} V_{0}^{-2} / F_{\psi_{i}}\left(\varphi_{0}\right)=A
$$

and the formula

$$
\psi_{i}=\psi_{i 0}+\frac{C_{i}}{V_{0} F_{\psi_{i}}\left(\varphi_{0}\right)} \ln \left|t_{E}-t_{0}\right|
$$

for modulus and axion fields. By taking into account (4.8) and (4.3) in the neighborhood of this solution we receive

$$
\phi-\phi_{0} \sim \cos \left(\frac{\sqrt{\omega_{0}}}{V_{0}} \ln \left|t_{E}-t_{0}\right|\right)
$$

At tree level taking into account the expressions (4.17) for dilaton we find from (4.21)

$$
\begin{aligned}
e^{2 \varphi} & =\frac{A_{1}}{2}\left(\left|t_{E}-t_{0}\right|^{\alpha_{1}}+\frac{\alpha_{2}}{A_{1}^{2}}\left|t_{E}-t_{0}\right|^{-\alpha_{1}}\right) \\
\alpha_{1} & = \pm\left[\left(A-\sum_{i} C_{i}^{2} V_{0}^{-2}\right)(n-1)\right]^{1 / 2}, \quad \alpha_{2}=2 C_{h}^{2}(n-1) / \alpha_{1}^{2} V_{0}^{2},
\end{aligned}
$$

$A_{1}$ - is an integration constant (for the type-I superstring one has $e^{\varphi}$ in the left handside and $\alpha_{1} / 2$ in the right hand side). For nonzero antisymmetric tensor field from (4.26) one obtains a restriction on possible values of dilaton field: $e^{4 \varphi} \geq \alpha_{2}$. At the same approximation from the equations (4.22) we obtain

$$
\begin{aligned}
\psi_{i} & =\psi_{i 0}+\frac{C_{i}}{V_{0}} \ln \left|t_{E}-t_{0}\right| \\
h & =h_{0}-\frac{4 C_{h}}{\alpha_{1} V_{0} A_{1}^{2}}\left[\left|t_{E}-t_{0}\right|^{2 \alpha_{1}}+\alpha_{2} / A_{1}^{2}\right]^{-1}
\end{aligned}
$$


with integration constants $\psi_{i 0}$ and $h_{0}$. The corresponding solutions in string frame can be found by transformation (4.11). Here we shall consider a simple case of zero Kalb - Ramond field, corresponding to the value of constant $\alpha_{2}=0\left(C_{h}=0\right)$. At tree level the synchronous time coordinates in string and $\mathrm{E}$ - frames are related by

$$
t_{s}-t_{s 0}=\text { const } \cdot\left|t_{E}-t_{0}\right|^{1+\alpha_{1} /(n-1)}
$$

and the solutions of string frame are

$$
\begin{aligned}
\widetilde{R}_{i} & \sim\left|t_{s}-t_{s 0}\right|^{\widetilde{H}_{i 0}}, \quad e^{2 \varphi} \sim\left|t_{s}-t_{s 0}\right|^{\widetilde{\alpha}_{1}} \\
e^{\psi_{i}} & \sim\left|t_{s}-t_{s 0}\right|^{\psi_{i 1}}, \quad \sum_{i=1}^{p} n_{i} \widetilde{H}_{i 0}=1+\widetilde{\alpha}_{1}, \quad \sum_{i=1}^{p} n_{i} \widetilde{H}_{i 0}^{2}+\sum_{i} \psi_{i 1}^{2}=1,
\end{aligned}
$$

where the constants can be expressed via the previously defined constants by relations

$$
\begin{aligned}
\widetilde{\alpha}_{1} & =\frac{\alpha_{1}}{1+\alpha_{1} /(n-1)}, \quad \psi_{i 1}=\frac{\widetilde{\alpha}_{1} C_{i}}{\alpha_{1} V_{0}} \\
\widetilde{H}_{i 0} & =\left(H_{i 0}+\frac{\alpha_{1}}{n-1}\right) \frac{\widetilde{\alpha}_{1}}{\alpha_{1}}
\end{aligned}
$$

For the pure gravi - dilaton case we have $\psi_{i 1}=0$ and (4.28) coincides with (4.12).

\section{$5 \quad$ Isotropic models with curved space}

In the previous section we have considered multidimensional anisotropic cosmological model with scalar and Kalb - Ramond fields for the case of Ricci - flat subspaces. We shall now consider the isotropic models with curved space. In E - frame the corresponding system of cosmological equations can be obtained from (4.1):

$$
\begin{aligned}
\dot{H}+H(n H-\dot{N} / N) & =N^{2} b_{1} \varepsilon-N^{2} k \frac{n-1}{R^{2}} \\
\ddot{\phi}+\dot{\phi}(n H-\dot{N} / N) & =\frac{1}{2} N^{2} \alpha_{\phi} \varepsilon,
\end{aligned}
$$

where

$$
b_{1}=\frac{1-a_{1}}{2(n-1)}, \quad a_{1}=\frac{p_{1}}{\varepsilon}
$$

The constraint equation has the form

$$
N^{2} \varepsilon+\dot{\phi}^{2}=n(n-1)\left(H^{2}+k N^{2} / R^{2}\right)
$$

The solution of cosmological equations becomes essentially simple for a source with the extreme hard equation of state $\varepsilon=p_{1}\left(a_{1}=1\right)$. In particular, as it was shown in the previous section, this condition is fulfilled for a set of scalar fields and Kalb 
- Ramond field with the Lagrangian (4.14). For such sources the first equation of system (5.1) coincides with the corresponding vacuum equation and is solved most simply in terms of conformal time $\eta$, corresponding to the gauge $N=R$ :

$$
d s^{2}=R^{2}\left(d \eta^{2}-d l^{2}\right) .
$$

Now by integrating the first equation of (5.1) for Hubble function we find

$$
H^{2}=B / R^{2(n-1)}-k
$$

where $B$ is an integration constant. It follows from here, that for closed models $(k=1)$ scale factor varies in finite interval $0 \leq R \leq R_{m}$, where $R_{m}=B^{1 / 2(n-1)}$. Making use of Eq. (5.5) from the constraint equation one obtains

$$
N^{2} \varepsilon+\left(\frac{d \phi}{d \eta}\right)^{2}=\frac{n(n-1)}{R^{2(n-1)}} B
$$

and therefore if the energy density is nonnegative, then $B \geq 0$. Note, that the solutions with negative $B$ can be realized only for models with spaces of negative curvature, and in this case the scale factor varies within limits

$$
|B|^{1 / 2(n-1)} \equiv R_{\min } \leq R<\infty
$$

and the corresponding models are nonsingular:

$$
R=R_{\min }[\operatorname{ch}(n-1) \eta]^{1 /(n-1)} .
$$

For the solutions with non-negative energy density, solving the equation (5.5) for the scale factor we have

$$
R=R_{m}\left|\frac{1}{\sqrt{k}} \sin [\sqrt{k}(n-1) \eta]\right|^{1 /(n-1)}
$$

In view of this from the constraint equation we find for energy density

$$
N^{2} \varepsilon+\left(\frac{d \phi}{d \eta}\right)^{2}=\frac{k n(n-1)}{\sin ^{2}[\sqrt{k}(n-1) \eta]} .
$$

In what follows we shall assume $0 \leq(n-1) \eta \leq \pi$ for $k=1$ and $0 \leq(n-1) \eta<\infty$ for $k=0,-1$. The solutions in the other regions can be obtained fro here. Let us consider as a nongravitational source the system with the Lagrangian density (4.14) and energy density (4.15). Below we shall not specify the value of dimension $n$ of space. The substitution (4.15) into the constraint equation (5.10) leads to the following equation

$$
\frac{d \phi}{d \eta}=\frac{ \pm \sqrt{k}}{\sin [\sqrt{k}(n-1) \eta]}\left[n(n-1)-R_{m}^{2(1-n)} \sum_{i}^{\prime} \frac{C_{i}^{2}}{F_{\psi_{i}}}\right]^{1 / 2}
$$


By integrating this equation for dilaton field we find the following relation

$$
\begin{aligned}
\pm \ln \left|\frac{1}{\sqrt{k}} \operatorname{tg}\left[\sqrt{k}(n-1) \frac{\eta}{2}\right]\right|= & (n-1) \int\left[\frac{n}{n-1}\left(\frac{\widetilde{F}_{R}^{\prime}}{\widetilde{F}_{R}}\right)^{2}-\frac{4 \widetilde{F}_{\varphi}}{\widetilde{F}_{R}}\right]^{1 / 2} \\
& \times\left[n(n-1)-R_{m}^{2(1-n)} \sum_{i} C_{i}^{2} \frac{\widetilde{F}_{R}}{\widetilde{F}_{\psi_{i}}}\right]^{-1 / 2} d \varphi
\end{aligned}
$$

For given functions $\widetilde{F}_{K}(\varphi)$ in the Lagrangian (2.1) this formula together with (5.9) determines the dynamics of corresponding cosmological model. For models with negative energy density the evolution of dilaton field is determined from the relation

$$
\pm 2 \operatorname{arctg}\left[e^{(n-1) \eta}\right]=(n-1) \int\left[\sum_{i}^{\prime} \frac{C_{i}^{2}}{F_{\psi_{i}}} R_{\min }^{2(1-n)}-n(n-1)\right]^{-1 / 2} d \phi
$$

and the scale factor has the form (5.8). Further we shall consider models with nonnegative energy density. For given function $\varphi(\eta)$, determined from (5.12), the dependence of the other fields on time is determined from the equation (3.25):

$$
\frac{d \psi_{i}}{d \eta}=\frac{C_{i}}{R_{m}^{n-1}} \frac{\sqrt{k} F_{\psi_{i}}^{-1}(\varphi)}{\sin [\sqrt{k}(n-1) \eta]} .
$$

In particular, for $\psi_{i}=h$ one obtains the equation for the axion field. For the given coupling functions, the expressions (5.9), (5.12), (5.14) determine the evolution of isotropic cosmological models with $k=0, \pm 1$ in terms of conformal time coordinate $\eta$.

In what follows it proves convenient to define the new variable $z$ according to

$$
z=\left|\frac{1}{\sqrt{k}} \operatorname{tg}[\sqrt{k}(n-1) \eta / 2]\right| .
$$

The formula (5.9) for the scale factor can be now written down as

$$
R=R_{0}\left(\frac{z}{1+k z^{2}}\right)^{1 /(n-1)} .
$$

The above mentioned solutions are written through conformal time $\eta$, which is connected to E - frame synchronous time coordinate by relation

$$
t_{E}-t_{0}= \pm \int R d \eta= \pm \frac{2 R_{0}}{n-1} \int_{0}^{z} \frac{z^{1 /(n-1)} d z}{\left(1+k z^{2}\right)^{n /(n-1)}},
$$

where the integral in the right hand side can be expressed through hypergeometric function. The corresponding solutions in string frame can be found by transformation 
(4.11). For open models $(k=-1)$ the variable $z$ varies within limits $0 \leq z \leq 1$, thus, as it follows from (5.17), time coordinate is not limited. In the case of closed models $0 \leq z<\infty$, and $t_{E}$ varies in finite limits:

$$
\left|t_{E}-t_{0}\right| \leq t_{e m} \equiv \frac{R_{0}}{n-1} B\left(\frac{n}{2(n-1)}, \frac{n}{2(n-1)}\right),
$$

where $B(x, y)$ - is Euler $\beta$-function. For the models with a flat space from (5.16) we have $R \sim\left|t_{E}-t_{0}\right|^{1 / n}$, which is the isotropic limit of the solution (4.8). In the limit $t_{E} \rightarrow t_{0}$ the contribution of curvature terms is negligible and the behavior of open and closed models is the same as in a flat case. For an open model and $t_{E} \rightarrow \infty$ from (5.16) and (5.17) we have $R \sim t_{E}$.

For the solutions with constant dilaton $\varphi=\varphi_{0}$ the value $\varphi_{0}$ has to be a solution of the equation (4.5) and from (5.11) we obtain the following relation between integration constants

$$
\sum_{i}^{\prime} \frac{C_{i}^{2}}{F_{\psi_{i}}\left(\varphi_{0}\right)} R_{m}^{2(1-n)}=n(n-1)
$$

and the expression

$$
\psi_{i}=\frac{C_{i}}{(n-1) F_{\psi_{i}}\left(\varphi_{0}\right)} R_{m}^{1-n} \ln z+\text { const }
$$

for modulus and axion fields. In the neighborhood of this solution for models with variable dilaton one obtains $\phi-\phi_{0} \sim \cos \left(\sqrt{\omega_{0}} R_{m}^{1-n} \frac{\ln z}{n-1}\right)$.

We now turn to the consideration of the obtained solutions at tree level of the heterotic string ( the corresponding type-I solutions can be obtained by replacements $\left.e^{2 \varphi} \rightarrow e^{\varphi}, \beta_{1} \rightarrow \beta_{1} / 2\right)$, when the corresponding $\mathrm{E}$ - frame functions are defined by relations (4.17). The integration of (5.14) now leads to the result

$$
\psi_{i}=\psi_{i 1} \ln z+\psi_{i 0}, \quad \psi_{i 1}=\frac{C_{i}}{(n-1) R_{m}^{n-1}} .
$$

Similarly, from the relation (5.12) for dilaton field we find

$$
e^{2 \varphi}=\left(A_{1} z^{\beta_{1}}+\beta_{2} z^{-\beta_{1}} / A_{1}\right) / 2,
$$

where $A_{1}$ is an integration constant, and $\beta_{i}$ are connected to the earlier introduced constants by relations

$$
\begin{aligned}
& \beta_{1}= \pm\left[n-R_{m}^{2(1-n)} \sum_{i} C_{i}^{2} /(n-1)\right]^{1 / 2}, \\
& \beta_{2}=2 C_{h}^{2} /\left[n(n-1) R_{m}^{2(n-1)}-\sum_{i} C_{i}^{2}\right] .
\end{aligned}
$$

As it follows from (5.22) for models with nonzero Kalb - Ramond field the values of dilaton are limited by inequality $e^{4 \varphi} \geq \beta_{2}$. From here as a necessary condition of 
existence of weak coupling region we obtain: $\beta_{2} \ll 1$. By taking into account the tree level expression $F_{h}=e^{4 \varphi} / 2$ from the equation (5.14) we find for axion

$$
h=h_{0}-\frac{4 C_{h}}{(n-1) \beta_{1} R_{m}^{n-1}}\left(A_{1}^{2} z^{2 \beta_{1}}+\beta_{2}\right)^{-1}
$$

with a new integration constant $h_{0}$. For the scale factor and synchronous time coordinate $t_{s}$ of string frame from the relation (4.11) we have

$$
\begin{aligned}
\widetilde{R} & =\widetilde{R}_{0}\left(z \frac{z^{\beta_{1}}+\beta_{2} z^{-\beta_{1}} / A_{1}^{2}}{1+k z^{2}}\right)^{1 /(n-1)} \\
t_{s} & =t_{s 0}+\frac{ \pm 2}{n-1} \int_{0}^{z} \frac{\widetilde{R}(z) d z}{1+k z^{2}} .
\end{aligned}
$$

As in the $\mathrm{E}$ - frame, here time coordinate $t_{s}$ varies in finite limits for closed models and is unlimited for $k=0,-1$. For the models with zero scalar fields $\psi_{i}$ the constant $\beta_{1}= \pm \sqrt{n}$ and from (5.16), (5.22) and (5.25) we obtain the solutions earlier considered in 45 .

In the limit $z \rightarrow 0$ all solutions tend to the solution with a flat space, and in the E - frame

$$
R \sim\left|t_{E}-t_{0}\right|^{1 / n}, \quad e^{2 \varphi} \sim\left|t_{E}-t_{0}\right|^{\beta_{3} \frac{n-1}{n}}, \quad t_{E} \rightarrow t_{0}
$$

$\left(\beta_{3}=-\left|\beta_{1}\right|\right.$ for $\beta_{2} \neq 0$ and $\beta_{3}=\beta_{1}$ for $\left.\beta_{2}=0\right)$. In the string frame one obtains

$$
\widetilde{R} \sim\left|t_{s}-t_{s 0}\right|^{\frac{1+\beta_{3}}{n+\beta_{3}}},\left|t_{s}-t_{s 0}\right|=\left|t_{E}-t_{0}\right|^{\frac{n+\beta_{3}}{n}}, t_{s} \rightarrow t_{s 0}
$$

In this limit one has $R \rightarrow 0$, while the limiting value of string frame scale factor is zero or infinity for $\beta_{3}>-1$ and $\beta_{3}<-1$, correspondingly. Note, that $\left|\beta_{3}\right|=\left|\beta_{1}\right| \leq \sqrt{n}$. For the case $\beta_{3}=-1$ one has

$$
\widetilde{R} \sim \text { const }+\left(t_{s}-t_{s 0}\right)^{2}, \quad e^{2 \varphi} \sim\left|t_{s}-t_{s 0}\right|^{-1}, \quad t_{s} \rightarrow t_{s 0}
$$

Despite the fact that in this case the string frame scale factor is finite at $t_{s}=t_{0}$ the model is singular, because at that point the dilaton field is divergent. Similar conclusion can be reached by starting directly with the expression for scalar curvature, which in virtue of (5.5) can be presented in the form

$$
R_{\text {curv }}=\frac{n(n-1) B}{R^{2 n}} \sim\left(t_{E}-t_{0}\right)^{-2} \sim\left|t_{s}-t_{s 0}\right|^{\frac{-2 n}{n-1}}, \quad t_{s} \rightarrow t_{s 0}
$$

We turn now to the other asymptotic limit $t_{s} \rightarrow \infty$, which is possible for open and flat models only. From the relations (5.22) and(5.25) one can easily finds

$$
\begin{aligned}
& \widetilde{R} \sim t_{s}^{\frac{1-\beta_{3}}{n-1}}, \quad e^{2 \varphi} \sim t_{s}^{\frac{\beta_{3}(n-1)}{\beta_{3}-n}}, \quad t_{s} \rightarrow \infty, \quad k=0 \\
& \widetilde{R} \sim t_{s}, \quad e^{2 \varphi} \sim\left(A_{1}+\beta_{2} / A_{1}\right) / 2+1 / t_{s}^{n-1}, \quad t_{s} \rightarrow \infty, \quad k=-1
\end{aligned}
$$


Note that for $k=-1$ the limiting value $\varphi(\infty)>\varphi_{\min }=\ln \beta_{2} / 4$. For the relative contribution of moduli and antisymmetric tensor field to the energy density one obtains

$$
\frac{n(n-1) B \varepsilon}{\dot{\phi}^{2} R^{2}+\varepsilon}=\sum_{i} C_{i}^{2}+2 C_{h}^{2} e^{-4 \varphi}
$$

and the evolution of the universe is moduli dominated at $t_{s} \rightarrow t_{s 0}$. The string frame scale factor $\tilde{R}$ and dilaton $e^{\varphi}$ (as functions of $t_{s}$ ) in spatially flat, open and closed $n=3$ models are plotted in Fig.1 for $\left|\beta_{1}\right|<1$ (left hand-side) and $\left|\beta_{1}\right|>1$ (right hand-side). The plots for the case $\left|\beta_{1}\right|=1$ can be found in [29] (see below).

For $n=3$ by introducing a new time coordinate $t$ in accordance with

$$
2 k t^{2}=t_{+}^{2}+k t_{-}^{2}-\left(t_{+}^{2}-k t_{-}^{2}\right) \cos 2 \sqrt{k} \eta, \quad z=\sqrt{\frac{t^{2}-t_{-}^{2}}{t_{+}^{2}-k t^{2}}}
$$

and choosing $R_{m}^{2}=\left(t_{+}^{2}-k t_{-}^{2}\right) / 2$ for the line element corresponding to the solution (5.9) we can write

$$
d s^{2}=\frac{t^{2} d t^{2}}{\sqrt{\left(t_{+}^{2}-k t^{2}\right)\left(t^{2}-t_{-}^{2}\right)}}-\sqrt{\left(t_{+}^{2}-k t^{2}\right)\left(t^{2}-t_{-}^{2}\right)} d l^{2} .
$$

For the particular values of integration constants

$$
\psi_{i 1}=-1, \quad\left(\Rightarrow \beta_{1}=1\right)
$$

from the expressions (5.21), (5.22) and (5.24) we have

$$
\begin{aligned}
e^{2 \varphi} & =\frac{t^{2}\left(1-k t_{-}^{2} / t_{+}^{2}\right)}{2 \sqrt{\left(t_{+}^{2}-k t^{2}\right)\left(t^{2}-t_{-}^{2}\right)}}, \quad e^{2\left(\psi_{i}-\psi_{i 0}\right)}=\frac{t_{+}^{2}-k t^{2}}{t^{2}-t_{-}^{2}} \\
h & =h_{0} \pm \frac{2 t_{+} t_{-}}{t_{+}^{2}-k t_{-}^{2}}\left(\frac{t_{+}^{2}}{t^{2}}-k\right)
\end{aligned}
$$

where the substitution $\beta_{2}=t_{-}^{2} / t_{+}^{2}$ is used. The expressions (5.33), (5.35) represent $4 D$ tree level solutions of the effective string theory in the $\mathrm{E}$ - frame. The corresponding solutions in the string frame can be found by transformation (4.11) with the function $\widetilde{F}_{R}=e^{-2 \varphi}$. They were found earlier and are investigated in Ref. [29] by using the $5 D$ solutions of [74, 75].

\section{Conclusion}

In the present paper homogeneous anisotropic cosmological models are investigated within the framework of the effective string gravity with higher genus corrections 
and described by the action (2.1). The various conformal frames are considered, the most important of which are the string, Einstein and Jordan frames. A class of nongravitational sources is distinguished, for which the Jordan frame can be realized. The field equations are derived in general conformal frame. It allows to obtain the dynamics of model in various conformal frames by the corresponding choice of the conformal factor. The equations of the cosmological model are the simplest in the $\mathrm{E}$ - frame. In the absence of nongravitational sources the solutions with Ricci - flat subspaces have the form (4.8), (4.10) in the E - frame and (4.12) in the string frame. They generalize the multidimensional Kasner solution in GR. Further as an additional source a set of modulus and Kalb - Ramond fields is considered. In the case of zero potential terms this system is reduced to an effective source with the extreme hard equation of state. For such a source the equation of the dilaton field can be presented in the form (4.4). The phase trajectories of the corresponding dynamical system in the neighborhood of the solutions with constant dilaton are closed curves and the nonmonotonic dilaton dependence of the coupling functions can not stabilize the expectation value of the dilaton. This is a consequence of the fact that for the source under consideration the corresponding friction term in dilaton field equation vanishes. In the E- frame the corresponding solution with a set of modulus and antisymmetric tensor fields as a nongravitational source the scale factors coincide with those for vacuum solution. For anisotropic models with Ricci - flat subspaces and for given coupling functions in the Lagrangian (2.1) the evolution of dilaton, modulus and axion fields is determined by relations (4.21), (4.22). Section 5 is devoted to the isotropic models with a space of arbitrary curvature and with a set of modulus fields and Kalb - Ramond field as an additional source. The corresponding solutions have the most simple form in terms of conformal time. The $\mathrm{E}$ - frame scale factor, dilaton, modulus fields and axion are determined by relations (5.9), (5.12) and (4.20), and E frame synchronous time coordinate is connected with the conformal time by relation (5.17). Unlike the flat and open models, lifetime of closed models is finite (see (5.18)). The considered solutions are singular at some finite time moment. In a vicinity of this point all solutions tend to the solution with Ricci - flat subspaces. The tree level limit of received solutions is considered. The corresponding formulas have the form (5.21), (5.22), (5.24) in the E - frame and (5.25) in the string frame and are generalizations of the earlier known solutions [29, 34].

For the solutions considered above the growth of the curvature is unbounded. In the higher curvature regime string corrections modify the lowest - order effective action (2.1). In particular, higher derivative terms, whose contribution is controlled by the string tension $\alpha^{\prime}$, become important. The investigations have shown such corrections can regularize the curvature singularity [37], [39] - 433.

\section{Acknowledgments}

The work is supported in part by Grant 96-855 of Ministry of Science and Education of the Republic of Armenia. I would like to thank Prof. R. Brustein for bringing the references [36, 37] to my attention. 


\section{References}

[1] J. H. Schwarz, Nucl. Phys. Proc. Suppl. B55 (1997) 1( hep-th/9607067) and hep-th/9607201.

[2] J. Polchinski, Rev. Mod. Phys. 68 (1996) 1245.

[3] R. Dijkgraaf, Les Houches Lectures on Fields, Strings and Duality, hepth/9703136.

[4] I. Antoniadis, H. Partouche and T. R. Taylor, "Lectures on Heterotic - Type I Duality", preprint CERN-TH 97-136 (hep-th/9706211).

[5] W. Lerche, "Recent Developments in String Theory", preprint CERN-TH/97-299 (hep-th/9710246).

[6] E. Witten, Nucl. Phys. B443 (1995) 85 .

[7] T. Banks, Nucl. Phys. Proc. Suppl. B41 (1995) 21.

[8] R. R. Khuri, Helv. Phys. Acta 67 (1994) 884.

[9] M. J. Duff, R. R. Khuri and J. X. Lu, Phys. Rep. 259 (1995) 213.

[10] A. Strominger and C. Vafa, Phys. Lett. B379 (1996) 99.

[11] P. Kanti and K. Tamvakis, Phys. Lett. B352 (1997) 30.

[12] P. Binetruy and M. K. Gaillard, Phys. Rev. D34 (1996) 3069.

[13] J. Ellis, K. Enqvist, D. V. Nanopoulos and M. Quiros, Nucl. Phys. B277 (1986) 231.

[14] R. C. Myers, Phys. Lett. B199 (1987) 371.

[15] I. Antoniadis, C. Bachas, J. Ellis and D. V. Nanopoulos, Phys. Lett. B211 (1998) 393 ; Nucl. Phys. B328 (1989) 117.

[16] R. Brandenberger and C. Vafa, Nucl. Phys. B316 (1989) 391.

[17] M. Mueller, Nucl. Phys. B337 (1990) 37.

[18] G. Veneziano, Phys. Lett. B265 (1991) 287.

[19] A. A. Tseytlin, Mod. Phys. Lett. A6 (1991) 1721. 
[20] B. A. Campbell, A. Linde and K. A. Olive, Nucl. Phys. B355 (1991) 146.

[21] M. C. Bento, O. Bertolami and P. M. Sa, Phys. Lett. B262 (1991) 11.

[22] J. A. Casas, J. Garcia-Bellido and M. Quiros, Nucl. Phys. B361 (1991) 713 .

[23] A. A. Tseytlin, Class. Quantum Grav. 9 (1992) 979 .

[24] A. A. Tseytlin, Int. J. Mod. Phys. D1 (1992) 223.

[25] A. A. Tseytlin and C. Vafa, Nucl. Phys. 372 (1992) 443.

[26] J. Garcia-Bellido and M. Quiros, Nucl. Phys. B368 (1992) 463 .

[27] M. Gasperini and G. Veneziano, Astropart. Phys. 1 (1993) 317 ; Phys. Rev. D50 (1994) 2519 .

[28] M. Gasperini and G. Veneziano, Mod. Phys. Lett. A8 (1993) 3701.

[29] K. Behrndt and S. Forste, Nucl. Phys. B430 (1994) 441.

[30] J. Levin and K. Freese, Nucl. Phys. B421 (1994) 635 .

[31] T. Damour and A. M. Polyakov, Nucl. Phys. B423 (1994) 532 .

[32] E. Raiten, Nucl. Phys B416 (1994) 881.

[33] R. Brustein and G. Veneziano, Phys. Lett. B277 (1994) 256.

[34] N. Caloper, R. Madden and K. A. Olive, Nucl. Phys. B452 (1995) 677 ; Phys. Lett. B371 (1996) 34 .

[35] R. Easther, K. Maeda and D. Wands, Phys. Rev. D53 (1996) 4247.

[36] R. Brustein and R. Madden, "Graceful Exit and Energy Conditions in String Cosmology", preprint BGU-PH-97/06 (hep-th/9702043).

[37] R. Brustein and R. Madden, "A Model of Graceful Exit in String Cosmology", preprint BGU-PH-97/11 (hep-th/9708046).

[38] A. A. Saharian, "On Graceful Exit in String Cosmology with Pre-Big Bang Phase", hep-th/9709118.

[39] I. A. Antoniadis, J. Rizos and K. Tamvakis, Nucl. Phys. B415 (1993) 497.

[40] J. Rizos and K. Tamvakis, Phys. Lett. B326 (1994) 57 .

[41] R. Easther and K. Maeda, Phys. Rev. D54 (1996) 7252 . 
[42] S. J. Rey, Phys. Rev. Lett. 77 (1996) 1929.

[43] M. Gasperini and G. Veneziano, Phys. Lett. B387 (1996) 715 .

[44] J. Levin, Phys. Rev. D51 (1995) 1536 .

[45] E. J. Copeland, A. Lahiri and D. Wands, Phys. Rev. D50 (1994) 4868 ; D51 (1995)1569 .

[46] A.A.Saharian, Astrophysics 38 (1995) 101.

[47] A.A.Saharian, Astrophysics 38 (1995) 291 ; 38 (1995) 448 ; 40 (1997) 233 ; 40 (1997).

[48] A.A.Saharian, Astrophysics 39 (1996) 279 .

[49] M. C. Bento and O. Bertolami, Class. Quantum Grav. 12 (1995) 1919.

[50] M. Gasperini, "Relic Dilatons in String Cosmology", preprint CERN-TH/96-330 (gr-qc/9611059).

[51] H. Lu, S. Mukherji, C. N. Pope and K.-W.Xu, Phys. Rev. D55 (1997) 7926.

[52] M. Gasperini, J. Maharana and G. Veneziano, Nucl. Phys. B472 (1996) 349 .

[53] T. Damour and A. Vilenkin, Phys. Rev. D53 (1996) 2981.

[54] R. Poppe and S. Schwager, Phys. Lett. B393 (1997) 51.

[55] J. D. Barrow and K. E. Kunze, Phys. Rev. D55 (1997) 623 .

[56] K. A. Meissner, Phys. Lett. 392 (1997) 298.

[57] N. Kaloper and K. A. Meissner, "Duality Beyond the First Loop", preprint CERN-TH/97-113 (hep-th/9705193).

[58] A. Lukas, B. A. Ovrut and D. Waldram, Phys. Lett. B393 (1997) 65 ; UPR-723T (hep-th/7610238).

[59] N. Kaloper, Phys. Rev. D55 (1997) 3394 .

[60] H. Lu, S. Mukherji and C. N. Poppe, Phys. Rev. D55 (1997) 7926.

[61] A. Buannano, K. A. Meissner, G. Ungarelli and G. Veneziano, "Classical Inhomogeneities in String Cosmology", preprint CERN-TH/97-124 hep-th/9706221.

[62] M. Gasperini, M. Maggiore and G. Veneziano, Nucl. Phys. B494 (1997) 315.

[63] M. S. Turner and E. J. Weinberg, Phys. Rev. D56 (1997) 4604. 
[64] M.Green, J. Schwarz and E. Witten, Superstring theory, v. 1, 2. Cambridge University Press, Cambridge, 1987.

[65] E. Kiritsis, Introduction to Superstring Theory, preprint CERN-TH/97-218 (hepth/9709062).

[66] C. Lovelace, Phys. Lett. B135 (1984) 75 .

[67] E. S. Fradkin and A. A. Tseytlin, Phys. Lett. B158 (1985) 316 ; Nucl. Phys. B261 (1985) 1 .

[68] C. G. Callan, D. Friedan, E. J. Martinec and M. J. Perry, Nucl. Phys. B262 (1985) 593 .

[69] D. Gross and J. Sloan, Nucl. Phys. B291 (1987) 41.

[70] E. Kiritsis, C. Kounnas, P. M. Petropoulos and J. Rizos, Nucl. Phys. B483 (1997) 141.

[71] I. L. Shapiro and H. Tanaka, Phys. Rev. D52 (1995) 2162 .

[72] C. M. Will, Theory and Experiment in Gravitational Physics. Cambridge University Press, Cambridge, 1993.

[73] T. Damour and K. Nordvedt, Phys. Rev. D58 (1993) 3436 .

[74] G. W. Gibbons and K. Maeda, Nucl. Phys. B298 (1988) 741.

[75] T. Horowitz and A. Strominger, Phys. Rev. Lett. 61 (1991) 2930 . 


\section{Figure Captions}

Fig.1. Plots for the string frame scale factor $\widetilde{R}$ (solid lines) and dilaton $e^{\varphi}$ (dashed)as functions of synchronous time coordinate $t_{s}$ in spatially flat $(\mathrm{a}, \mathrm{b})$, open $(\mathrm{c}, \mathrm{d})$ and closed (e,f) $n=3$ models described by the solution (5.22), (5.25). On the left hand-side is $\left|\beta_{1}\right|<1$ and on the right hand-side is $\left|\beta_{1}\right|>1$. 